\title{
Large Scale Electric Car Sharing Stimulates EV Adoption and EVSE Infrastructure Deployment
}

\author{
Hervé Muller \\ President, BlueIndy; VP Blue Solutions (Bolloré)
}

\begin{abstract}
Summary
After operating Autolib' in Paris since 2011, Bolloré has now successfully launched in North America with a first all-electric car sharing service in Indianapolis named BlueIndy. The service will allow the City to gain up to 1,000 public charge points (EVSEs) and 500 Bluecar BEVs. The service represents a new mode of electric mobility and the ability for all in Indianapolis to experience EVs.
\end{abstract}

\section{BlueIndy History}

The presentation will cover:

- The history of the BlueIndy service, a first in North America

- The contractual background

- The role of the City

- The role of the electric utility

- Other key stakeholders in the community

\section{BlueIndy Statistics}

The presentation will cover:

- The size of the service

- The adoption by the population

- Usage numbers, duration, etc

- Demographics served

\section{BlueIndy Impact}

The presentation will cover:

- Members' needs

- Change in driving habits

- Benefits of the service

- Indianapolis transit options

\section{Applicability to other Cities}

The presentation will cover:

- Success criteria

- Key requirements

- How to successfully plan and deploy a similar service 


\section{Authors}

Hervé Muller, Vice President, Blue Solutions (Bolloré Group), President, BlueIndy.

Hervé Muller leads the introduction of Bolloré's Blue Solutions energy storage and electric mobility solutions in North America. Blue Solutions operates the world's largest EV car sharing service in Paris, France. Hervé is the president of BlueIndy, the new all-electric car sharing service launched in Indianapolis, which aims to deploy $500 \mathrm{EVs}$ and up to 1,000 charging stations. Hervé is also in charge of IER INC, another Bolloré company in the US and Canada. IER is a leader in airline passenger processing technology with solutions deployed at most of the world's airports. Hervé has previously managed sales, marketing, operations and project management for other leading technology providers and systems integrators, including NCR, SITA and ARINC.

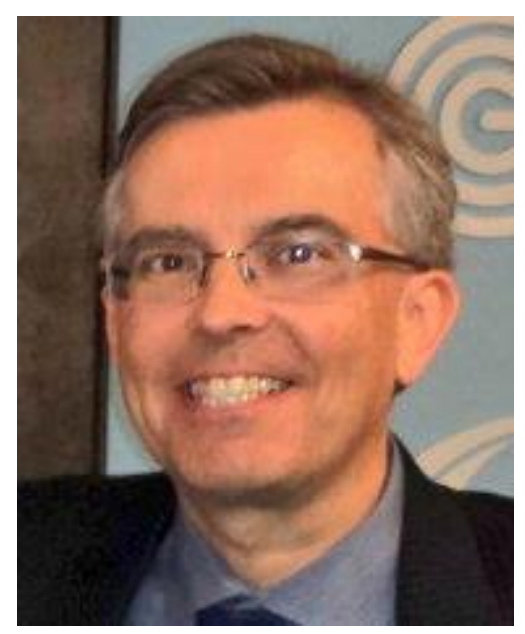

